\title{
Numerical Approach to Compute the Power Flow Solution of Hybrid Generation, Transmission and Distribution Systems
}

\author{
F. Bizzarri, Senior Member, IEEE, D. del Giudice, Student Member, IEEE, D. Linaro, and A. Brambilla Member, \\ IEEE
}

\begin{abstract}
We consider the formulation of hybrid three-phase, three-sequence models of distribution systems and single-phase, three-sequence models of generation and transmission systems to compute the power flow solution. The term hybrid refers to formulations of modern power systems that mix electronic equipment such as HVDC links and that consider converter-connected loads and generators with conventional electro-mechanical elements such as for example synchronous generators and turbine governors. The target of hybrid formulations is to allow the simulation of the entire mixed power system. In this paper we specifically consider the computation of the power flow solution of hybrid power systems. We propose a systematic approach to correctly link different models and to compute the power flow solution with an acceptable accuracy level.
\end{abstract}

\section{INTRODUCTION}

The increasing usage of power generation and transmission equipments connected to the grid through interfacing switching converters, such as clean energy sources (e.g., wind farms or photovoltaic plants) and HVDC links, leads to difficulties in computing the power-flow (PF) solution without resorting to simplified models of these systems. On the distribution side, as described in [1], a new model of load was introduced that accounts for single phase induction motor, incandescent, halogen, fluorescent lamps with electronic ballast, computers, electronic loads. Most of these loads lead to unbalanced distribution systems polluted by high frequency harmonics.

The need of simulating complex and hybrid unbalanced power systems (PSs) triggered the development of new simulation tools for both the determination of the PF solution and the transient stability. New algorithms have been developed based on hybrid approaches. In [2], [3] the transmission system is modeled with a three-sequence formulation and the distribution system is modeled with the three-phase one. This choice is done with the target of achieving a good compromise between the numerical efficiency and accuracy of the analysis. In [4] an extended three-sequence formulation of the PF problem is provided through the re-introduction of the well-known modified nodal analysis.

Those hybrid approaches, based on the straightforward coupling of the three-sequence or three-phase model of the

Federico Bizzarri, Davide del Giudice, Daniele Linaro and Angelo Brambilla are with DEIB, Politecnico di Milano, Milano, Italy. E-mail: \{federico.bizzarri, davide.delgiudice, daniele.linaro angelo.brambilla $\} @$ polimi.it

Federico Bizzarri is also with the Advanced Research Center on Electronic Systems E. De Castro (ARCES), University of Bologna, Italy. distribution system and of the three-sequence or single-phase models of the generation and transmission system, can be largely inadequate and inaccurate. We show that (i) singlephase models may be better exploited in simulating hybrid power systems and (ii) that using in substitution the threesequence approach does not necessarily improve the accuracy of the PF solution. In this context we propose a novel approach to compute the PF solution of a hybrid PS, whose level of accuracy is defined by a given indicator. To adequately link the single- and three-phase models we introduce a coupling element that we refer to as virtual connector. It plays the same role of the well-known pseudo-analog-to-digital and pseudo-digital-to-analog converters inserted during mixed analog/digital simulations to couple the analog and digital model paradigms of the circuit. The words pseudo and virtual refer to the fact that such components do not exist in the real design but are introduced as simulation expedients.

\section{Single-PHASE EQUIVALENT MODEL}

It is often thought that the single-phase model of the PS can handle only the positive sequence (see the comment on page 5 of [1]). What is referred to as single-phase equivalent model in the literature is the result of applying the Park transform to the three-phase representation in the time domain of a PS [5]. The Park transform is such that, if the solution of a balanced three-phase model exhibits a single-tone spectrum, it shifts this component in the frequency origin, i.e., it becomes the complex DC component. As an example, the Park transform applied to the $v_{a}(t)=E_{o} \cos (\Omega t), v_{b}(t)=E_{o} \cos (\Omega t-2 \pi / 3)$ and $v_{c}(t)=E_{o} \cos (\Omega t+2 \pi / 3)$ voltages provides a vector of three constant components. This has the great advantage of transforming a periodic solution in the time domain of the three-phase system to an equilibrium point in the DQ0-frame. The computation of the steady state solution of the three-phase system is thus speeded up, as well assessed by the methods devoted to the PF solution calculation [6].

The Park transform can also be applied without introducing any approximation to unbalanced three-phase components. However, this leads to a spectrum component in the DQ0frame at $-2 f_{0}$, which implies the appearance of the negative sequence. As a result, the PF solution in the DQ0-frame is no longer a DC solution but a periodic orbit. Therefore, the aforementioned great advantage in quickly determining the PF solution is completely lost since time domain waveforms must be computed instead of a constant solution. 
More in general, the single-phase equivalent model is capable of accurately representing positive and negative sequences of three-phase systems together with harmonics (for example due to polluting loads and switching converters) but these representations are time-varying functions.

\section{POWER FLOW SOLUTION}

The PF solution is the key to performing subsequent transient stability analysis since it provides the PS initial conditions. For example, once the PF solution is available, synchronous machines, turbine governors and voltage regulators can be initialised. According to Section II, this can be done since it is assumed that only the positive sequence component is present in the single-phase equivalent models, thus for example synchronous generators operate in a steady state condition where the mechanical and electrical torques balance each other leading to constant values. To grasp what happens if this strong assumption does not hold, consider the simplest PS model

$$
\begin{aligned}
& \dot{\delta}(t)-\Omega(\omega(t)-1)=0 \\
& H \dot{\omega}(t)+D(\omega(t)-1)-P_{m}+P_{e}(t)=0 \\
& P_{e}(t)-\sum_{k=a, b, c} \underbrace{E_{o}^{2} G_{k} \cos ^{2}\left(\Omega t+\delta(t)+\psi_{k}\right)}_{P_{k}(t)}=0,
\end{aligned}
$$

where a synchronous machine (first two equations) is coupled to a three-phase resistive load of conductance $G_{a, b, c}$ (last equation). The $P_{e}(t)$ electric power can be considered as the sum of the $P_{a}(t), P_{b}(t)$ and $P_{c}(t)$ phase modulated "signals". The phase modulating signal is $\delta(t)$. It is well known that, if $G_{a}=G_{b}=G_{c} \equiv G, P_{e}=\frac{3}{2} G E_{o}^{2}$ since each $P_{k}(t)$ term contributes to a spectrum in which all harmonics cancel except the DC component. Thus $P_{e}$ is constant, which implies that by choosing $P_{m}=P_{e}$ we have $\dot{\omega}(t)=0$ and $\dot{\delta}(t)=0$. As a consequence, by canceling all the components of the resulting spectrum but the DC, the $\delta(t)$ phase modulating function becomes a constant itself. Thus, in computing the PF solution, the dynamical model of the synchronous machines can be neglected since it does not participate in the PF solution. The $P_{m}$ value can be computed after the determination of the PF solution and the synchronous machine can be initialised after the determination of the PF solution. This leads to a great advantage in terms of numerical computation and it is what is conventionally done.

In an unbalanced load situation, for example with $G_{a} \neq$ 0 and $G_{b}=G_{c}=0$, or similarly with polluting harmonics, achieving $P_{e}=G_{a} E_{o}^{2} \cos ^{2}(\Omega t+\delta(t))$ and choosing $P_{m}=\frac{1}{2} G_{a} E_{o}^{2}$, i.e., the average value of $P_{e}$, fundamental and harmonic components do no longer cancel. In this case $\delta(t)$ can be a very complex function. To have some insight, let us initially and arbitrarily assume $\delta(t)=\beta / 2 \sin \left(\omega_{m} t\right)$. We have

$$
\begin{gathered}
P_{e}(t)=G_{a} E_{o}^{2} \cos ^{2}\left(\Omega t+\frac{\beta}{2} \sin \left(\omega_{m} t\right)\right) \\
=\frac{G_{a} E_{o}^{2}}{2}\left(1+\cos \left(2 \Omega t+\beta \sin \left(\omega_{m} t\right)\right)\right) \\
=\frac{G_{a} E_{o}^{2}}{2}\left(1+\sum_{n=-\infty}^{\infty} J_{n}(\beta) \cos \left(\left(2 \Omega+n \omega_{m}\right) t\right)\right),
\end{gathered}
$$

where $J_{n}(\cdot)$ are the Bessel functions of the first kind of order $n$ [7]. This clearly shows that the spectrum of $P_{e}(t)$ has an infinite number of harmonics. So has the spectrum of $\omega(t)$, which means that the turbine shaft undergoes undesired "vibrations" and sub-harmonics. The fact that $\omega(t)$ leads to an infinite spectrum obviously implies that $\delta(t)$ has an infinite spectrum too. Thus our initial assumption that $\delta(t)$ is a single tone sinusoidal function is an extreme and basically wrong simplification since we clearly come to the conclusion that $\delta(t)$ has an infinite spectrum. We remark that the relevant issue is that infinite spectra replace simple positive sequences represented by complex constant values only. We thus state that

- the solution of an unbalanced generation, transmission and distribution system is not an equilibrium point when computed either with the single-phase equivalent model or the three-sequence model. It cannot be correctly computed by any conventional PF solver and even by those proposed for example in [2], [4].

- The solution is a periodic orbit in the time domain both in the single-phase and three-sequence models.

\section{THE IEEE 9 BUS SYSTEM AS TEST CASE WITH AN UNBALANCED LOAD}

We use a modified version of the IEEE 9 bus system ${ }^{1}$ as test case to show how the constant PF solution transforms in a periodic solution. The $\mathrm{G} 2$ generator is equipped with an extra turbine governor and a turbine shaft model [6].

We first compute ${ }^{2}$ the PF solution of the conventional test system, which turns out to be stable, thus showing that there cannot be the onset of any oscillation. However, this holds provided that no perturbations given by unbalanced components, regardless of their magnitude, are introduced.

To prove this statement, we connected an extra three-phase resistive load at BUS5 absorbing $10 \mathrm{MW}$ at nominal voltage, modeling an unbalanced portion of a distribution system. We perform an electro-magnetic transient simulation of the full system, starting from the previously obtained PF solution, to determine the steady state periodic behaviour of the unbalanced system. Although the connection of the unbalanced load alters the total load power by only $3.1 \%$, it introduces oscillations and harmonics in the entire PS. This is clearly visible in Fig. 1, where the spectra of the $P_{e_{1}}, P_{e_{2}}$ and $P_{e_{3}}$ active power of the G1, G2 and G3 synchronous generators, respectively, are shown. Note that the unbalanced load excites also sub-synchronous resonating modes of the shaft of $\mathrm{G} 2$, i.e., the only generator with a turbine shaft model. These oscillations and their frequency beats propagate to the entire PS affecting G1 and G3. The resonance of the G2 shaft is marked by the increase of the magnitude of power spectra in the $[1,6] \mathrm{Hz}$ interval. Harmonics are marked by peaks in the spectra at several multiples of $60 \mathrm{~Hz}$. These results clearly show that the conventional PF computation is fully inadequate when unbalanced power systems are considered.

\footnotetext{
${ }^{1}$ Due to space reasons we do not show the schematic of the IEEE 9 bus power system. It is a well known benchmark largely used in the literature. Its schematic can be easily found.

${ }^{2}$ All the simulations were performed through the PAN simulator [8], [9].
} 


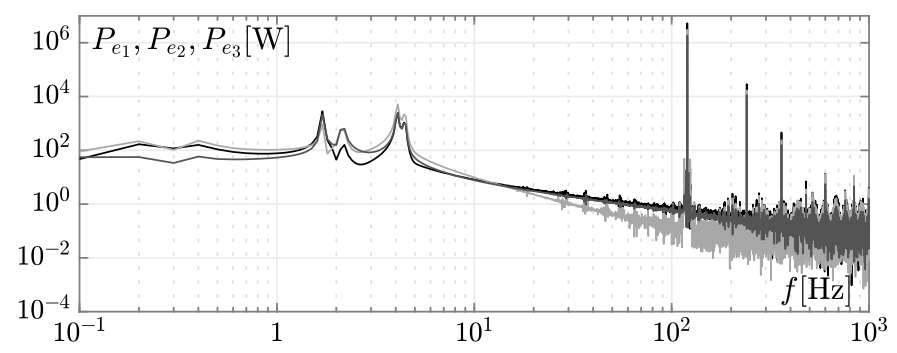

Figure 1. The spectrum of the active power of G1, G2 and G3 synchronous generators $(\log -\log$ plot). Frequency resolution is $0.1 \mathrm{~Hz}$. The DC component is not shown. x-axis: frequency [Hz]. y-axis: $P_{e_{1}}, P_{e_{2}}, P_{e_{3}}$ from black to lighter gray $[\mathrm{W}]$.

\section{THE PROPOSED APPROACH}

We consider once more the IEEE 9 bus system, modeled as a single-phase system, and connect a three-phase nonlinear load to BUS5 through the virtual connector mentioned in the Introduction. A hybrid power system is thus obtained. We expect the steady state we are looking for to be composed of two coexisting sub-solutions, i.e., the constant one related to the single-phase model and the periodic time domain solution of the three-phase nonlinear load.

The virtual connector (VC) exchanges power between the single-phase and three-phase systems. In principle the $\mathrm{VC}$ should be nonenergic (or inactive), meaning that it should not alter the above-mentioned power exchange through additional power injection or absorption. The schematic of the $\mathrm{VC}$ is shown in Fig. 2. The star connection of the controlled voltage sources ${ }^{3}$ in the three-phase section of the VC is such that $\imath_{a, b, c}^{\mathrm{VC}}$ corresponding to the $\imath_{d, q}^{\mathrm{VC}}$ currents of the controlled sources in the single-phase section lead to a null homopolar component in the DQ0-frame. This is guaranteed by imposing

$$
\left[\begin{array}{c}
l_{d}^{\mathrm{VC}} \\
\imath_{q}^{\mathrm{vc}} \\
0
\end{array}\right]=\kappa \overbrace{\left[\begin{array}{ccc}
\cos (\Omega t) & \cos \left(\Omega t-\frac{2 \pi}{3}\right) & \cos \left(\Omega t+\frac{2 \pi}{3}\right) \\
-\sin (\Omega t) & -\sin \left(\Omega t-\frac{2 \pi}{3}\right) & -\sin \left(\Omega t+\frac{2 \pi}{3}\right) \\
\sqrt{1 / 2} & \sqrt{1 / 2} & \sqrt{1 / 2}
\end{array}\right]}^{\mathbf{P}}\left[\begin{array}{c}
\imath_{a}^{\mathrm{vC}} \\
\imath_{b}^{\mathrm{vc}} \\
\imath_{c}^{\mathrm{vc}}
\end{array}\right],
$$

where the $\mathbf{P}$ matrix represents the Park transform, $\kappa=\sqrt{\frac{2}{3}}$, and $\Omega=2 \pi f_{o}$ [5]. The $f_{o}$ frequency is that of the overall power system network, i.e., $50 \mathrm{~Hz}$ or $60 \mathrm{~Hz}$. The sub-matrix obtained by removing the last row of $\mathbf{P}$ is referred to as $\widehat{\mathbf{P}}$. Being $v_{d, q}^{\mathrm{VC}}$ the voltages at the bus in the single-phase section of the $\mathrm{VC}$, the $v_{a, b, c}^{\mathrm{VC}}$ voltages are derived as

$$
\left[\begin{array}{c}
v_{a}^{\mathrm{VC}} \\
v_{b}^{\mathrm{vC}} \\
v_{c}^{\mathrm{vC}}
\end{array}\right]=\sqrt{\frac{2}{3}} \widehat{\mathbf{P}}^{\mathrm{T}}\left[\begin{array}{l}
\hat{v}_{d}^{\mathrm{vC}} \\
\hat{v}_{q}^{\mathrm{VC}}
\end{array}\right]
$$

The peculiar aspect of the $\mathrm{VC}$ is that the bus to which its single-phase section is connected is fed by the $\hat{\imath}_{d, q}^{\mathrm{VC}}$ currents, which are given by

$$
\hat{\imath}_{d}^{\mathrm{VC}}=\frac{\Omega}{2 \pi} \int_{t_{0}}^{t_{0}+\frac{2 \pi}{\Omega}} \imath_{d}^{\mathrm{VC}}(\tau) d \tau, \hat{\imath}_{q}^{\mathrm{VC}}=\frac{\Omega}{2 \pi} \int_{t_{0}}^{t_{0}+\frac{2 \pi}{\Omega}} \imath_{q}^{\mathrm{VC}}(\tau) d \tau .
$$

\footnotetext{
${ }^{3}$ We use the modified nodal analysis formulation that allows an easy implementation of the characteristic of the controlled sources [4].
}

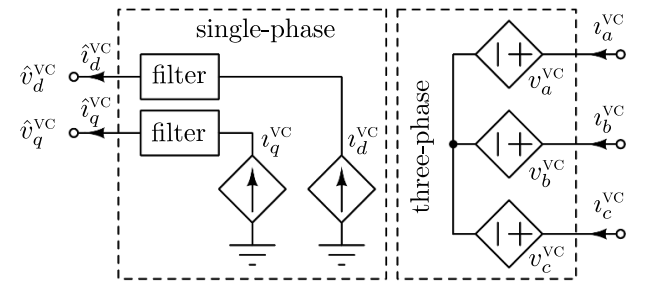

Figure 2. The schematic of the VC.

The $\hat{\imath}_{d, q}^{\mathrm{vC}}$ currents are computed by applying a filter to $\imath_{d, q}^{\mathrm{VC}}$ that extracts only the DC components. Filtering is mandatory since as shown in Section IV the spectra of currents of the threephase model of the complex part of the PS may be richer than a simple tone at $\Omega / 2 \pi$. By using the $\hat{\imath}_{d, q}^{\mathrm{VC}}$ filtered component, it is thus guaranteed that, in computing the solution of the overall PS, bus voltages and powers at the single-phase side are constant and satisfy the conventional paradigm used in computing the PF solution.

The filtering action makes the $\mathrm{VC}$ dissipative, potentially altering the PF solution of the hybrid PS. In order to take this into account, we introduce the following accuracy index

$$
\mathcal{E}_{\mathrm{hd}}^{j}=\frac{\widetilde{I}_{j, 0} \widetilde{I}_{j, 0}^{*}}{\sum_{k=-K}^{K} \widetilde{I}_{j, k} \widetilde{I}_{j, k}^{*}},
$$

where $\widetilde{I}_{j}$ are the components of the complex spectrum of the currents of all the $N$ vCs organised in the $\widetilde{\imath}_{j}=\left(\imath_{d, j}^{\mathrm{VC}}, \imath_{q, j}^{\mathrm{VC}} \mathrm{I}\right) \in$ $\mathbb{C}$ vector, computed by the complex Fast-Fourier transform, $\mathrm{I}=\sqrt{-1}$ and $j=1, \ldots, N . k=0$ indexes the DC component and $K$ is the maximum harmonic in the spectrum (bilateral). The $j$-th $0 \leq \mathcal{E}_{\text {hd }}^{j} \leq 1$ gives an idea on how much power lays at the other components of the spectrum excluding the DC, that gives the magnitude of the positive sequence. The higher $\mathcal{E}_{\text {hd }}^{j}$, the lower the power dissipated by the $j$-th $\mathrm{VC}$ with respect to the transferred one and, thus, the more accurate the solution.

The solution of the full PS is determined by simultaneously computing the coupled periodic solution of the three-phase sub-system with for example the shooting method [10] and the constant solution of the single-phase sub-system. Coupling is done at VC level by balancing (Kirchhoff Current Law) the currents injected in each $\mathrm{VC}$ from the power bus and the $\hat{\imath}_{d, q}^{\mathrm{VC}}$ currents determined through filtering as described in (2).

\section{THREE-PHASE RECTIFIERS}

To show how the solution of a hybrid power system is computed, we once again employ a modified version of the IEEE 9 bus system. The schematic of the three-phase rectifier used as nonlinear load is shown in the dashed box of Fig. 3. At first the $r, s, t$ terminals are connected to the $a, b, c$ ones of the $\mathrm{VC}$, i.e., the filter outside the dashed-box is not considered. The $d$ and $q$ terminals of the VC are connected to BUS5 of the IEEE 9 bus system. $R_{O}$ is the loading resistor supplied by the rectified voltage filtered by the $C_{o}$ capacitor. The application of the proposed method shows that the power flowing through the VC is $P=8.47 \mathrm{MW}, Q=4.03 \mathrm{MVAR}$. The active power of the slack generator increases from $71.641 \mathrm{MW}$ (no rectifier 


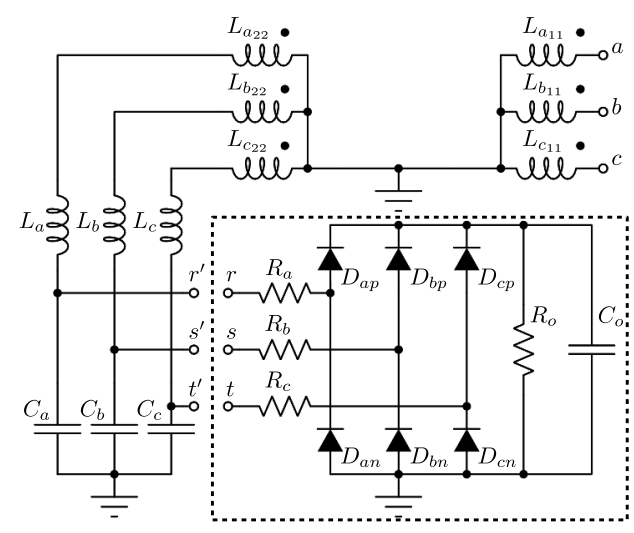

Figure 3. In the dashed box a three-phase rectifier is represented. $R_{a}=R_{b}=$ $R_{c}=10 \mathrm{~m} \Omega, R_{o}=25 \Omega, C_{o}=10 \mu \mathrm{F}$. Outside the dashed box, a passive filter is represented used to improve the three-phase rectifier connecting the $r-r^{\prime}, s-s^{\prime}$ and $t-t^{\prime}$ pairs of terminals. $L_{a}=L_{b}=L_{c}=50 \mathrm{mH}$, $C_{a}=C_{b}=C_{c}=30 \mu \mathrm{F}$, the step-down transformer is ideal and lowers voltage by 20 times.

connected) to $80.118 \mathrm{MW}$. The voltage magnitude of BUS5 slightly increases due to the reactive power injected by the rectifier. The main waveforms of the rectifiers are shown in Fig. 4. The distorted line currents, the ON/OFF action of the diodes and their effect on the line currents are clearly visible. In this case, the accuracy index is $\mathcal{E}_{\mathrm{hd}}=0.425$ which shows that too much power should be injected to BUS5 at high frequencies. The low $\mathcal{E}_{\text {hd }}$ tells that results obtained through hybrid simulation may be unreliable. The insertion of the VC must be moved in a section of the hybrid system where filtering is active (the dynamic model of some transmission lines should be considered). Note that in practice it is highly undesirable (and against directives on power system quality of service) to inject harmonics in the power transmission and distribution system. Thus, instead of moving the $\mathrm{VC}$, we insert the passive filter shown in Fig. 3 that we previously neglected by connecting the $r-r^{\prime}, s-s^{\prime}$ and $t-t^{\prime}$ pairs of terminals and removing the connection to ground of the negative DC terminal of the rectifier. The filter insertion mitigates the unwanted current distortion of the rectifier. The obtained results, depicted once more in Fig. 4, show a noticeable reduction in current distortion. This leads to $\mathcal{E}_{\text {hd }}=0.941$, which may be acceptable. The power flowing through the $\mathrm{VC}$ is now $P=4.7 \mathrm{MW}$ and $Q=5.2 \mathrm{kVAR}$, whereas the active power of the slack generator lowers to $76.542 \mathrm{MW}$.

\section{A. The modified Nordic test system}

The rectifier example described in the previous section may be considered a "toy circuit": we thus chose as an example of a more complex power system the NORDIC32, to show how the solution of an hybrid system is computed. A full description of the NORDIC 32 system with values of parameters of loads, lines, generators, shunts, the block schematics of automatic voltage regulators, turbine governors and under load tap changers can be found in [11], [12]. The NORDIC32 system becomes unstable due to the progressive action of the under load tap changers when the line that connects the BUS-4032
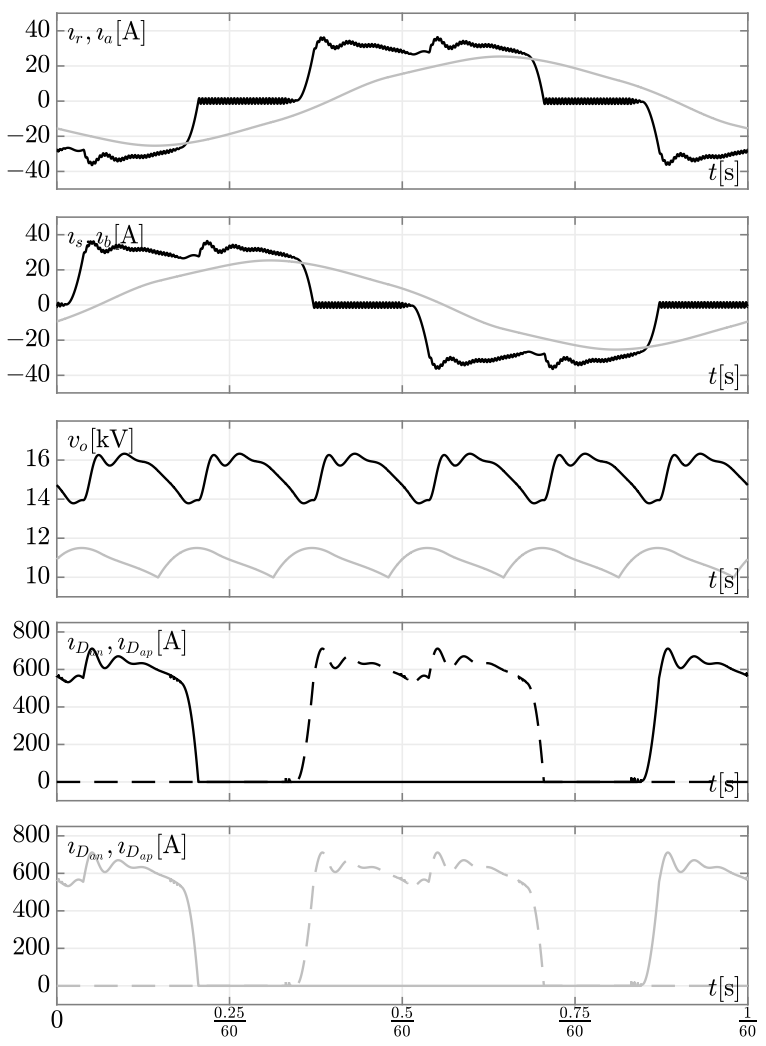

Figure 4. In black the main waveforms from the rectifier circuit shown in Fig. 3 (a-case) and in gray those of the rectifier with the filter (b-case). From top: panel 1: $r$ line current (a-case) and $a$ line current (b-case); panel 2: $s$ line current (a-case) and $b$ line current (b-case); panel 3: voltages across $R_{o}$ (both cases); panel 4: currents through the $D_{a n}$ (solid) and $D_{a p}$ (dashed) diodes (a-case); panel 5: currents through the $D_{a n}$ (solid) and $D_{a p}$ (dashed) diodes (b-case).

with BUS-4044 is opened after a fault. In order to improve the power transfer capability of the transmission system when the fault occurs, we modified the NORDIC 32 system by adding an high voltage direct current (HVDC) link between BUS-4011 (sender) and BUS-4045 (receiver). In these busses, VCs were used to connect the HVDC link to the power system.

The schematic of the modified NORDIC32 power system is shown in Fig. 5. The sender voltage source converter (VSC) regulates the power injected in the HVDC link; the receiver VSC regulates the DC voltage. The two VSCs inject null reactive power. We use detailed models of both VSC converters; they work with a PWM waveform at $213 f_{0}$, where $f_{0}$ is the locking frequency of the PLLs (practically $50 \mathrm{~Hz}$ ). More details on the VSC converters, their controllers and the LCL filters can be found in [13]. We performed hybrid power-flow analyses with two different working conditions of the HVDC link. The former was performed by setting the power of the sender at $500 \mathrm{MW}$ and the latter by setting it at $1.2 \mathrm{GW}$. The former leads to a stable system while the latter leads to an unstable system (in the neighborhood of stability boundary). A time domain analysis starting from the computed power-flow solution confirms that this modified version of the NORDIC 32 hybrid system is unstable. In Fig. 6 we show some waveforms of the HVDC system related to the unstable solution. Recall that 


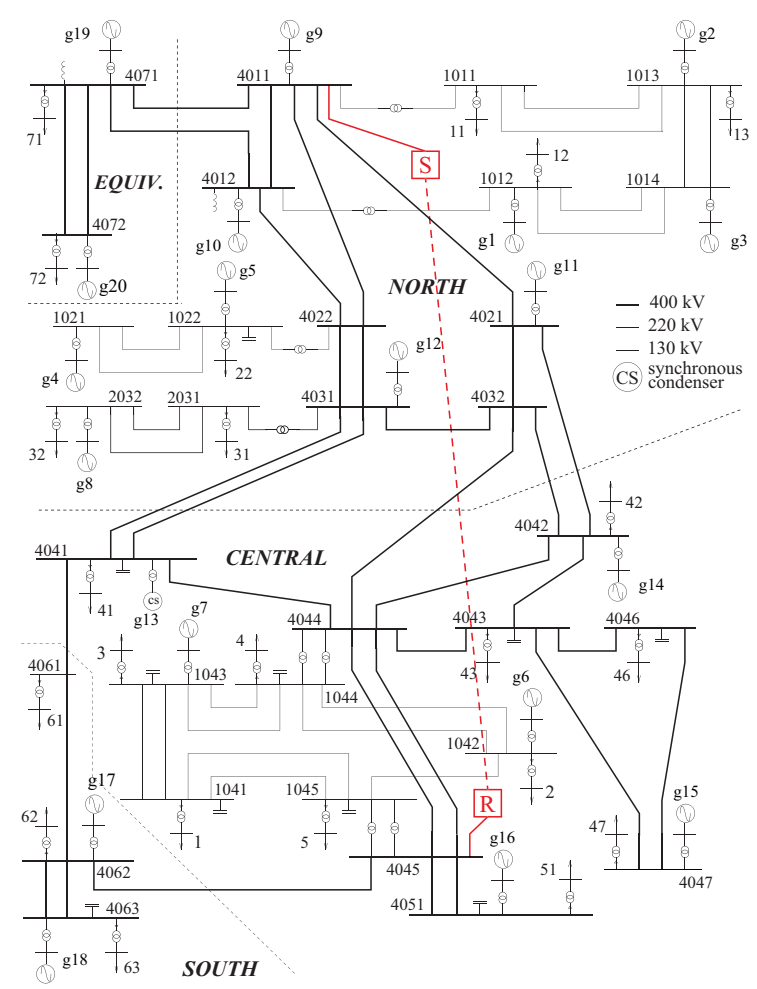

Figure 5. The one-line schematic of the Nordic test system with the addition of a HVDC link between BUS-4011 and BUS-4045 (dashed red line connecting the boxes labeled with S and R). The parameters of the LCL filter used in the VSC are inductances equal to $1.8 \mathrm{mH}$ and capacitances equal to $27 \mu \mathrm{H}$.

the hybrid power-flow solutions leads to constant waveforms along one working period of the single phase equivalent model of the power system and to periodic waveforms of the threephase detailed model of the power system and HVDC link. By simple inspection of the waveforms in Fig. 6 we see that the three-phase line currents of the sender are reasonable and that the current ripple of the HVDC link is simulated in detail. These waveforms are peculiar of the proposed numerical method and we underline once more that the NORDIC32 power system, the VSCs and HVDC link were simulated without resorting to any simplification or equivalent reduced model. The accuracy indices are $\mathcal{E}_{\text {hd }}^{\text {bus-4045 }}=0.9995$ and $\mathcal{E}_{\text {hd }}^{\text {bus-4011 }}=0.9998$. They are largely acceptable, i.e. the hybrid power-flow solution has an adequate accuracy.

\section{DISCUSSION AND CONCLUSIONS}

We have shown how harmonic pollution caused by unbalanced loads does not allow computing the PF solution in a conventional way. This hinders the correct initialization of power system components for a subsequent transient stability analysis. We presented the $\mathrm{VC}$, a pseudo-element that couples the single-phase and three-phase models of an hybrid power system. By doing so, the PF solution is composed of constant and periodic functions, related to the single- and three-phase models, respectively. We have shown the features of the proposed method by computing the PF solution of a modified version of the IEEE 9 bus and of the NORDIC 32 power systems.

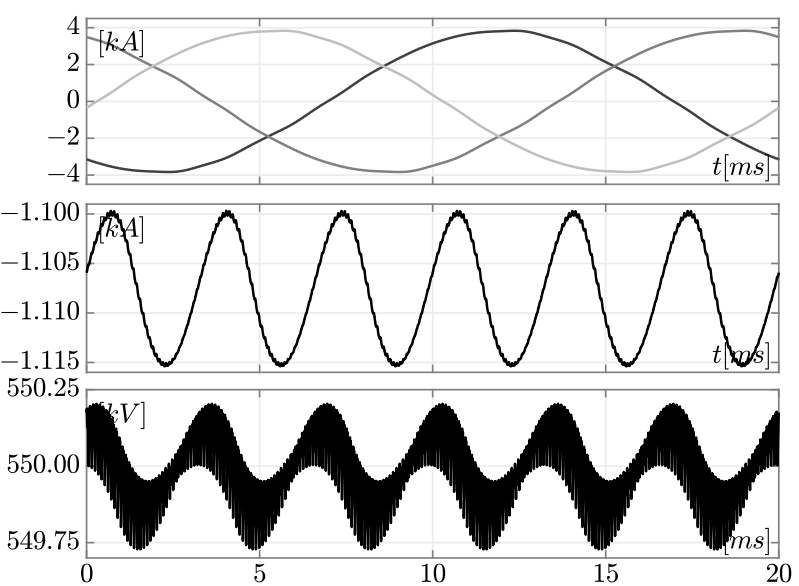

Figure 6. From top: panel 1: line currents of the sender VSC, showing an acceptable sinusoidal shape; panel 2: HVDC current; panel 3: voltage of the positive DC line, the ripple due to the switching of VSC converter is visible.

\section{REFERENCES}

[1] D. Kosterev, A. Meklin, J. Undrill, B. Lesieutre, W. Price, D. Chassin, R. Bravo, and S. Yang, "Load modeling in power system studies: Wecc progress update," in 2008 IEEE/PES General Meeting - Conversion and Delivery of Electrical Energy in the 21st Century, July 2008, pp. 1-8.

[2] Q. Huang and V. Vittal, "Integrated transmission and distribution system power flow and dynamic simulation using mixed three-sequence/threephase modeling," IEEE Transactions on Power Systems, vol. 32, no. 5, pp. 3704-3714, Sept 2017.

[3] - , "Advanced emt and phasor-domain hybrid simulation with simulation mode switching capability for transmission and distribution systems," IEEE Transactions on Power Systems, vol. 33, no. 6, pp. 62986308, Nov 2018.

[4] I. Kocar, J. Mahseredjian, U. Karaagac, G. Soykan, and O. Saad, "Multiphase load-flow solution for large-scale distribution systems using MANA," in 2014 IEEE PES General Meeting - Conference Exposition, July 2014, pp. 1-1.

[5] P. Kundur, Power System Stability and Control, ser. The EPRI power system engineering series. McGraw-Hill, 1984.

[6] F. Milano, Power System Modelling and Scripting. London: Springer, 2010.

[7] A. Carlson and P. Crilly, Communication Systems: An Introduction to Signals and Noise in Electrical Communication, Fifth Edition, 5th ed. McGraw-Hill Higher Education, 2009.

[8] F. Bizzarri and A. Brambilla, "PAN and MPanSuite: Simulation vehicles towards the analysis and design of heterogeneous mixed electrical systems," in IEEE International Conference of New Generation of Circuits and Systems (NGCAS), Sept 2017, pp. 1-4.

[9] F. Bizzarri, A. Brambilla, G. Storti Gajani, and S. Banerjee, "Simulation of real world circuits: Extending conventional analysis methods to circuits described by heterogeneous languages," IEEE Circuits and Systems Magazine, vol. 14, no. 4, pp. 51-70, 2014.

[10] F. Bizzarri, A. Brambilla, and F. Milano, "The probe-insertion technique for the detection of limit cycles in power systems," IEEE Transactions on Circuits and Systems I: Regular Papers, vol. 63, no. 2, pp. 312-321, Feb. 2016.

[11] L. D. P. Ospina, A. F. Correa, and G. Lammert, "Implementation and validation of the nordic test system in digsilent powerfactory," in 2017 IEEE Manchester PowerTech, June 2017, pp. 1-6.

[12] R. Van Cutsem and L. Papangelis, "Description, modeling and simulation results of a test system for voltage stability analysis," University of Liègen, Belgium, November 2013.

[13] A. Houari, H. Renaudineau, J. P. Martin, B. Nahid-Mobarakeh, S. Pierfederici, and F. Meibody-Tabar, "Large-signal stabilization of ac grid supplying voltage-source converters with lcl-filters," IEEE Trans. on Industry Applications, vol. 51, no. 1, pp. 702-711, Jan 2015. 